\section{Case Reports in Ophthalmology}

Case Rep Ophthalmol 2017;8:510-514

\title{
Acute Endophthalmitis Caused by Leuconostoc spp. following Intravitreal Bevacizumab Injection
}

\author{
Supreet Singh $^{a} \quad$ Chirag V. Patel $^{b} \quad$ Kamal Kishore $^{c_{1} d}$ \\ ${ }^{a}$ Rutgers-New Jersey Medical School, Newark, NJ, USA; ${ }^{b}$ Capital Health Medical Center, \\ Hopewell, NJ, USA; ' $I$ Illinois Retina and Eye Associates, Peoria, IL, USA; ${ }^{\text {UUniversity of }}$ \\ Illinois College of Medicine, Peoria Campus, Peoria, IL, USA
}

\section{Keywords}

Endophthalmitis $\cdot$ Leuconostoc $\cdot$ Intravitreal injections $\cdot$ Bevacizumab

\begin{abstract}
We present a case of acute endophthalmitis caused by Leuconostoc spp. following intravitreal bevacizumab injection. An 86-year-old immunocompetent female developed acute endophthalmitis after intravitreal injection of bevacizumab for neovascular age-related macular degeneration. The patient presented with pain, visual acuity of hand motions, hypopyon, and dense vitritis $96 \mathrm{~h}$ after treatment. She was treated with vitreous and anterior chamber tap followed by intravitreal injections of $1 \mathrm{mg}$ vancomycin, $2.25 \mathrm{mg}$ ceftazidime, and $400 \mu \mathrm{g}$ dexamethasone. Cultures revealed growth of Leuconostoc spp., a genus of gram-positive bacteria that is inherently resistant to vancomycin. Due to persistent inflammation, pars plana vitrectomy (PPV) with intravitreal injection of $0.4 \mathrm{mg}$ amikacin was performed 16 days later, followed by resolution of endophthalmitis and return of vision to 20/40. In conclusion, the management of acute endophthalmitis caused by Leuconostoc spp., a gram-positive coccobacillus, can be particularly challenging due to its inherent resistance to vancomycin. PPV with intravitreal amikacin led to resolution of endophthalmitis. Our case expands the number of cases of endophthalmitis caused by Leuconostoc spp. and highlights the possibility of Leuconostoc-related endophthalmitis in an outpatient setting in an immunocompetent host.
\end{abstract}




\section{Case Reports in Ophthalmology}

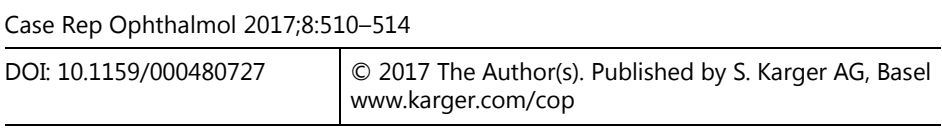

Singh et al.: Acute Endophthalmitis Caused by Leuconostoc spp. following Intravitreal Bevacizumab Injection

\section{Introduction}

Leuconostoc species are catalase-negative, gram-positive, facultatively anaerobic coccobacilli that may be misidentified as Enterococcus or Streptococcus species by routine biochemical testing [1]. The distinction among these bacteria is important because all clinical isolates of Leuconostoc possess a high level of resistance to vancomycin, with a minimal inhibitory concentration $>256 \mu \mathrm{g} / \mathrm{mL}$ [2]. Leuconostoc spp. are emerging pathogens responsible for a variety of infections including nosocomial outbreaks, particularly in patients immunocompromised due to neutropenia, cancer, or AIDS, those with severe burns, and in patients being treated with vancomycin [3-9]. To our knowledge, only 3 cases of Leuconostoc endophthalmitis, 2 acute and 1 chronic, have been reported so far [10-12]. We present a case of Leuconostoc-associated acute endophthalmitis in an outpatient setting following an intravitreal injection of bevacizumab in an 86-year-old immunocompetent patient who was successfully managed with pars plana vitrectomy (PPV) and intravitreal amikacin injection following failure of initial tap and inject procedure.

\section{Case Report}

An 86-year-old white female presented with pain, redness, and significant loss of vision in her left eye 4 days after receiving her first intravitreal injection of bevacizumab $(1.25 \mathrm{mg} / 0.05 \mathrm{~mL})$ for neovascular age-related macular degeneration in an outpatient officebased setting. Examination revealed visual acuity of hand motions. Intraocular pressure was $18 \mathrm{~mm}$ Hg. Slit lamp examination showed diffuse conjunctival congestion, marked corneal edema, 3-mm hypopyon, and fibrin in the anterior chamber (Fig. 1). Dilated fundus examination revealed dense vitritis with a barely visible optic nerve and no visible details of the retinal vessels or peripheral retina. Her past medical history was significant for gastroesophageal reflux disease, which was mild and longstanding and was not being treated with any medications at the time of presentation. She denied any history of recent hospitalization, antibiotic use, or immune suppression.

A diagnosis of acute endophthalmitis was made and a tap and inject procedure was performed. After we had obtained aqueous and vitreous specimens, the patient received intravitreal injections of vancomycin $(1 \mathrm{mg} / 0.1 \mathrm{~mL})$, ceftazidime $(2.25 \mathrm{mg} / 0.1 \mathrm{~mL})$, and dexamethasone $(400 \mu \mathrm{g} / 0.1 \mathrm{~mL})$. Culture results revealed growth of Leuconostoc. The patient continued to have visual acuity of hand motions and persistent hypopyon, along with dense vitritis. PPV followed by intravitreal injection of amikacin $(0.4 \mathrm{mg} / 0.1 \mathrm{~mL})$ was performed on postoperative day 16 . Her endophthalmitis rapidly resolved after vitrectomy and her visual acuity improved to $20 / 40$ at the 3-month postoperative visit, back to her level of vision prior to onset of the infection.

\section{Discussion}

Since the first reported human infection in 1985 [13], several cases and outbreaks of Leuconostoc infection have been reported, mostly in hospital settings and in immunocompromised patients [3-9]. Rare cases of Leuconostoc infection in immunocompetent hosts have been reported, including periprosthetic knee infection [14], and in a healthy infant [15]. To our knowledge, only 3 cases of endophthalmitis caused by Leuconostoc spp. have been 


\section{Case Reports in Ophthalmology}

reported, 1 after intravitreal injection of ranibizumab [12] and 2 after uncomplicated phacoemulsification $[10,11]$.

In the report by Damasceno et al. [12], the patient presented $48 \mathrm{~h}$ after uncomplicated intravitreal injection of ranibizumab. After vitreous biopsy, intravitreal injections of vancomycin and amikacin were administered. In addition, the patient was started on systemic gatifloxacin. Both cultures of blood and vitreous yielded Leuconostoc mesenteroides that was sensitive to ceftazidime, gentamicin, ciprofloxacin, amikacin, gatifloxacin, and moxifloxacin. Two additional intravitreal injections of amikacin, $48 \mathrm{~h}$ apart, were also administered. Despite treatment, the patient developed phthisis bulbi 2 months later. The patient had a history of cancer and chemotherapy, risk factors that could have potentially resulted in an increased susceptibility to the infection. As blood culture was also positive for Leuconostoc, the possibility of endogenous endophthalmitis cannot be completely ruled out. Furthermore, the injection was administered in a hospital-based setting instead of an office-based setting, a factor that has been related with nearly twice a higher rate of infection after intravitreal injection [16]. By contrast, our patient developed Leuconostoc-associated endophthalmitis after receiving an intravitreal injection in an office-based setting, was immunocompetent, and had a good outcome after PPV. The remaining 2 patients with Leuconostoc-associated endophthalmitis were immunocompetent and developed infection after uncomplicated phacoemulsification surgery [10,11]. The patient reported by Kumudhan and Mars [10], a 76-year-old healthy male, developed acute endophthalmitis 2 days after an uncomplicated phacoemulsification. Despite prompt intravitreal vancomycin and amikacin injections, the patient's final visual acuity was only hand motions. The patient reported by Durkin et al. [11] was a 43-year-old healthy male who developed chronic relapsing inflammation following an uncomplicated phacoemulsification that persisted despite a vitreous tap with injection of intravitreal vancomycin and ceftazidime. A vitrectomy procedure with repeat intravitreal injections of vancomycin and ceftazidime 8 months later yielded the growth of Leuconostoc and Olsenella uli and resulted in resolution of inflammation with return of vision to 6/12. Whether diminished immunity due to aging in our 86-year-old female patient predisposed her to Leuconostoc endophthalmitis is a matter of speculation. Our case report along with the 2 previously reported cases suggests the potential for Leuconostoc endophthalmitis in immunocompetent hosts.

The scarcity of reported Leuconostoc-based infections may also be attributed to the difficulty in isolating and identifying Leuconostoc through routine methods. Leuconostoc is often misidentified as Lactobacillus, Streptococcus, Pediococcus, Enterococcus, or Lactococcus, and such misidentification of the species can lead to an underestimation of its true incidence rate [1]. Leuconostoc presents unique challenges due to its inherent resistance to vancomycin, the drug of choice for endophthalmitis caused by gram-positive organisms. However, multidrug resistance does not seem to occur in Leuconostoc, and most reported cases, including those with endophthalmitis, have been treated with conventional antibiotics. Despite the well-known risk of aminoglycoside-induced retinal toxicity, we elected to treat the patient with intravitreal amikacin, with its relative safety compared to other aminoglycosides such as gentamicin or tobramycin [17]. This case report highlights Leuconostoc-associated endophthalmitis following intravitreal injection of bevacizumab and its successful treatment with PPV and intravitreal amikacin injection. 


\section{Statement of Ethics}

All procedures were done in accordance with the tenets of the Helsinki Declaration of 1964, as revised in 2013. Institutional review board approval was not required for this retrospective study involving a single case report.

\section{Disclosure Statement}

The authors have no financial disclosures to make or proprietary interests to disclose. There are no conflicts of interest for any of the authors.

\section{References}

1 Facklam R, Elliott JA: Identification, classification, and clinical relevance of catalase-negative, grampositive cocci, excluding the streptococci and enterococci. Clin Microbiol Rev 1995;8:479-495.

-2 Florescu D, Hill L, Sudan D, et al: Leuconostoc bacteremia in pediatric patients with short bowel syndrome: case series and review. Pediatr Infect Dis J 2008;27:1013-1019.

3 Handwerger S, Horowitz H, Coburn K, et al: Infection due to Leuconostoc species: six cases and review. Rev Infect Dis 1990;12:602-610.

-4 Bernaldo de Quiros JC, Munoz P, Cercenado E, et al: Leuconostoc species as a cause of bacteremia: two case reports and a literature review. Eur J Clin Microbiol Infect Dis 1991;10:505-509.

-5 Ferrer S, de Miguel G, Domingo P, et al: Pulmonary infection due to Leuconostoc species in a patient with AIDS. Clin Infect Dis 1995;21:225-226.

-6 Jimenez-Mejias ME, Becerril B, Gomez-Cia T, et al: Bacteremia caused by Leuconostoc cremoris in a patient with severe burn injuries. Eur J Clin Microbiol Infect Dis 1997;16:533-535.

-7 Cappelli EA, Barros RR, Camello TC, et al: Leuconostoc pseudomesenteroides as a cause of nosocomial urinary tract infections. J Clin Microbiol 1999;37:4124-4126.

-8 Bou G, Luis Saleta J, Saez Nieto JA, et al: Nosocomial outbreaks caused by Leuconostoc mesenteroides subsp. mesenteroides. Emerg Infect Dis 2008;14:968-971.

-9 Lee MR, Huang YT, Lee PI, et al: Healthcare-associated bacteraemia caused by Leuconostoc species at a university hospital in Taiwan between 1995 and 2008. J Hosp Infect 2011;78:45-49.

10 Kumudhan D, Mars S: Leuconostoc mesenteroides as a cause of post-operative endophthalmitis - a case report. Eye (Lond) 2004;18:1023-1024.

11 Durkin SR, Badenoch PR, Lake S, et al: Chronic postoperative endophthalmitis with isolation of Leuconostoc and Olsenella uli in an indigenous man. Clin Exp Ophthalmol 2008;36:801-802.

-12 Damasceno NP, Horowitz SA, Damasceno EF: Leuconostoc as a cause of endophthalmitis postintravitreal injection of ranibizumab. Ocul Immunol Inflamm 2016;24:118-119.

13 Buu-Hoi A, Branger C, Acar JF: Vancomycin-resistant streptococci or Leuconostoc sp. Antimicrob Agents Chemother 1985;28:458-460.

14 Franco-Cendejas R, Colin-Castro CA, Hernandez-Duran M, et al: Leuconostoc mesenteroides periprosthetic knee infection, an unusual fastidious gram-positive bacteria: a case report. BMC Infect Dis 2017;17:227.

15 Casanova-Roman M, Rios J, Sanchez-Porto A, et al: Leuconostoc bacteremia in a healthy infant. Minerva Pediatr 2003;55:83-86.

16 Tabandeh H, Boscia F, Sborgia A, et al: Endophthalmitis associated with intravitreal injections: officebased setting and operating room setting. Retina 2014;34:18-23.

17 D’Amico DJ, Caspers-Velu L, Libert J, et al: Comparative toxicity of intravitreal aminoglycoside antibiotics. Am J Ophthalmol 1985;100:264-275. 
Case Reports in
Ophthalmology

Case Rep Ophthalmol 2017;8:510-514

DOI: $10.1159 / 000480727$

(C) 2017 The Author(s). Published by S. Karger AG, Basel www.karger.com/cop

Singh et al.: Acute Endophthalmitis Caused by Leuconostoc spp. following Intravitreal Bevacizumab Injection

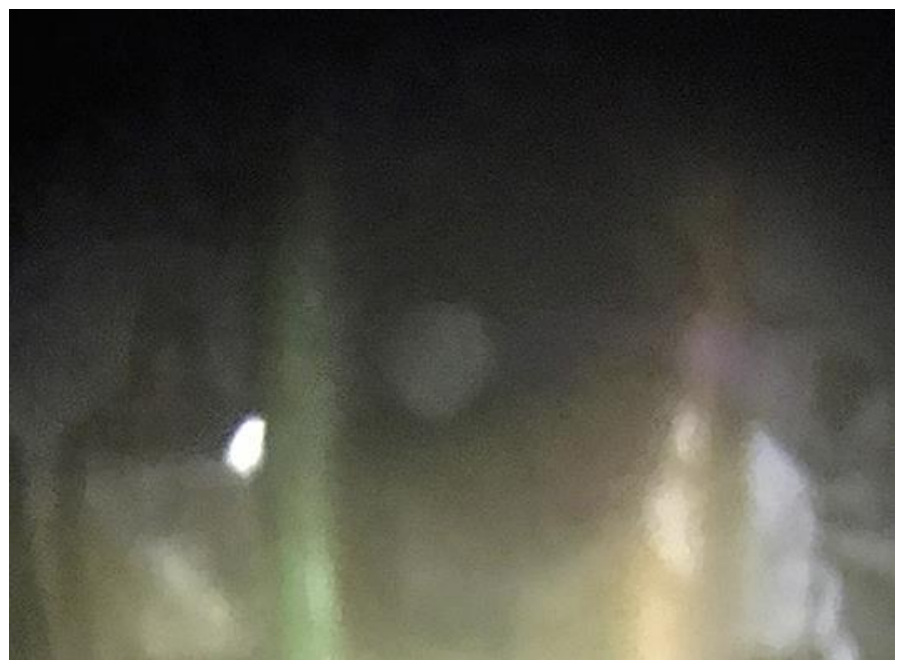

Fig. 1. Slit lamp photograph at presentation showing marked corneal edema, hypopyon, and fibrin membrane in the pupil. 